\title{
Non $k$-type behaviour of roughness when in-plane wavelength approaches the boundary layer thickness
}

\author{
B. Nugroho ${ }^{1}$, J. P. Monty ${ }^{1}$, I.K.A.P Utama ${ }^{2}$, \\ B. Ganapathisubramani ${ }^{3}$ and N. Hutchins ${ }^{1} \dagger$ \\ ${ }^{1}$ Department of Mechanical Engineering, The University of Melbourne, Victoria, 3010, \\ Australia \\ ${ }^{2}$ Department of Naval Architecture, Institut Teknologi Sepuluh Nopember, Surabaya, 60111, \\ Indonesia \\ ${ }^{3}$ Aerodynamics and Flight Mechanics Research Group, University of Southampton, \\ Southampton, SO17 1BJ, UK \\ (Received $\mathrm{xx}$; revised $\mathrm{xx}$; accepted $\mathrm{xx}$ )
}

A surface roughness from a recently cleaned and painted ship hull was scanned, scaled and replicated for laboratory testing to systematically investigate the influence of the ratio of in-plane roughness wavelength, $\lambda$, with respect to the boundary layer thickness $\delta$. The experiments were performed by geometrically scaling the surface which maintains a constant effective slope $E S_{x}$ and solidity $\Lambda$, while the ratio of $\lambda / \delta$ is varied. Here we scale the scanned roughness topography by a factor of 2.5 and 15 , and measure the mean velocity profiles in the turbulent boundary layers developing over these surfaces at a range of freestream velocities and streamwise measurement locations. The results show that the $2.5 \times$ scaled roughness, which has $\lambda / \delta \ll 1$ behaves in the expected $k$ type manner, with a roughness function $\Delta U^{+}$that is proportional to the viscous scaled roughness height. The $15 \times$ surface, however, which has $\lambda / \delta \approx 1$, exhibits very different non $k$-type behaviour. This larger surface does not approach the fully rough asymptote and also exhibits a drag penalty that is comparable to the $2.5 \times$ case despite the six-fold increase in the roughness height. Measurements on a spanwise/wall-normal plane reveal that the $15 \times$ surface has introduced a large scale spanwise variation in mean streamwise velocity (dispersive stresses) that extend far beyond the logarithmic region. Together this evidence suggests that a demarcation between $k$-type and non $k$-type behaviour can occur in situations where the in-plane roughness wavelength approaches the boundary layer thickness. This finding has important implications to how we scale small scale roughness from high $R e$ large-scale applications for testing in low $R e$ small-scale laboratory facilities or simulations.

\section{Introduction}

Turbulent wall-bounded flows over rough surfaces have a higher wall drag than a smooth counterpart, a measure of which is provided by the downward shift in the viscousscaled mean streamwise velocity profile in the logarithmic region. This shift is known as the Hama (1954) roughness function, $\Delta U^{+}=\Delta U / U_{\tau}\left(U_{\tau}=\sqrt{\tau_{w} / \rho}\right)$, where $U$ is the mean streamwise velocity, $U_{\tau}$ is skin friction velocity, $\tau_{w}$ is wall shear stress, and $\rho$ is fluid density. The magnitude of $\Delta U^{+}$is a function of the viscous scaled roughness height $k_{s}^{+}$, where $k_{s}$ is the equivalent sand grain roughness height and the ${ }^{+}$superscript indicates

$\dagger$ Email address for correspondence: nhu@unimelb.edu.au 
viscous scaling (i.e $k_{s}^{+}=k_{s} U_{\tau} / \nu$, where $\nu$ is kinematic viscosity). Although $k_{s}$ is a length, it is not a directly observable quantity from the roughness topography. Rather, it is a measure of the degree to which a surface roughness alters the near wall flow, and can only be obtained by applying fluid flow over the rough surface of interest, either experimentally or through simulation, at various Reynolds numbers (see Perry et al. 1969; Monty et al. 2016). The equivalent sandgrain roughness $k_{s}$ of a surface provides a corresponding equivalent grain size for a close-packed uniform sandgrain roughness (of the type used in the seminal experiments of Nikuradse 1933) which if exposed to the same flow in the fully-rough regime, would cause the same Hama roughness function $\Delta U^{+}$as the surface of interest.

An important concept in the study of rough wall-bounded turbulent flows is the assumption of Townsend's (1976) outer layer similarity hypothesis, which states that beyond the roughness sublayer and at a sufficiently large Reynolds number, turbulent motions are independent of the precise form of the surface roughness. Thus the roughness is 'felt' by the flow, only through a modified wall drag (and through the outer lengthscale). The existence of outer layer similarity can be identified from the collapse of mean velocity defect and outer-scaled turbulence intensity profiles between the rough surface and smooth surface (see for example Jiménez 2004; Flack et al. 2005; Schultz \& Flack 2005). The underlaying caveat from Townsend's outer layer similarity hypothesis is that the boundary layer thickness $\delta$ must be sufficiently large when compared to the 'extent of the flow patterns set up by the individual roughness elements' (Townsend 1976). Researchers generally interpret this as a requirement that the ratio $\delta / k$ must be large. For example both Jiménez (2004) and Flack et al. (2005) show that outer layer similarity is observed for $\delta / k_{s} \gtrsim 40$, while many other studies have observed outer layer similarity for much lower ratios of $\delta / k$ (for example Chan et al. 2015; Flack et al. 2007, 2020; Forooghi et al. 2018, 2017; Jelly \& Busse 2019). However, for the experiments conducted here, we will look at cases where $\delta \gg k$, conforming to these approximate limits, but where the ratio of in-plane roughness wavelength to boundary layer thickness $\lambda / \delta$ becomes large. Such scenarios are possible with surfaces that have low solidities or effective slopes. These surfaces are of interest, since there is a precedent in the literature demonstrating that the wall-normal 'extent of flow patterns' imposed by the roughness arrangement can become large relative to $\delta$ where the in-plane roughness lengthscale approaches $\delta$, limiting the observed outer layer similarity (see for example Nugroho et al. 2013; Chan et al. 2018; Chung et al. 2018; Medjnoun et al. 2018; Yang \& Anderson 2018; Anderson et al. 2018). Certainly an in-plane wavelength $\lambda$ that approaches $\delta$ will violate Townsend's (1976) assumptions of outer layer similarity (regardless of the value of $k / \delta$ ).

There are numerous instances in the literature where roughness with large ratios of in-plane wavelength to outer length-scale (large $\lambda / \delta$ ) have been investigated. Examples include Zilker et al. (1977); Angelis et al. (1997); Kruse et al. (2003); Hamed et al. (2015). When specifically assessed, the majority of these studies (Reda et al. 1974; Hudson et al. 1996; Nakagawa et al. 2003; Bhaganagar et al. 2004; Kruse et al. 2006; Sun et al. 2018) suggest that outer layer similarity is preserved over wavy surfaces. Few of these studies have explicitly addressed the influence of $\lambda / \delta$, with fewer still investigating any impact on $k$-type scaling. However, there are some noteworthy examples that are especially pertinent to the present investigation. Bhaganagar et al. (2004) studied the effect of $\lambda / \delta$, varying this ratio from $0.01 \leq \lambda_{y} / \delta \leq 0.5$ and from $0.04 \leq \lambda_{x} / \delta \leq 1.4$ at a single $k^{+}$, where $\lambda_{y}$ and $\lambda_{x}$ are the spanwise and streamwise in-plane wavelengths respectively. They found that while $\lambda_{y} / \delta$ had negligible effect on $\Delta U^{+}$, this ratio did affect the turbulence fluctuations in the outer layer, suggesting an effect on outer layer similarity. In some sense, the effect of large $\lambda / \delta$ on fluctuations in the outer layer is expected. Jiménez 
(2004) and Chan et al. (2018) have both discussed the proportionality between the roughness sublayer height and the in-plane roughness wavelength $\lambda$. When $\lambda / \delta$ is large, the roughness sublayer, and roughness induced secondary flows or dispersive motions, extend into the outer layer, affecting the measured turbulent statistics. Zenklusen et al. (2012) studied turbulent channel flow with a sinusoidal wavy bottom wall, and very large blockage ratios $k_{t} / H=0.1-0.3$, where $k_{t}$ is the peak-to-trough roughness height and $H$ is the total channel height. Since all of these surfaces had a fixed streamwise effective slope $\left(E S_{x}=0.2\right.$, defined as the mean absolute streamwise gradient of the surface) the ratio $\lambda / H$ for these surfaces was also large, ranging from $\lambda / H=1-3$. Hence, by every measure, these surfaces had roughness features that were large in comparison to the outer length-scale. The largest blockage case, which had the highest $\lambda / H$ differed from the other cases, exhibiting no separation at the roughness crest and with a modified large-scale turbulent structure (breakdown in outer layer similarity). A previous study by Kruse et al. (2006) had reported outer layer similarity for a surface with the same $E S_{x}$ but with smaller $k_{t} / H$ and $\lambda / H=1$. A further notable study on wavy surfaces is that by Nakato et al. (1985) who studied turbulent pipe flow over 2D approximately sinusoidal surfaces. They found that replicated painted surfaces with low effective slope $\left(E S_{x}<0.15\right)$ fail to reach the fully rough asymptote and exhibit non $k$-type behaviour. Though $\lambda / \delta$ was not considered as contributing to this behaviour, the largest wavelengths investigated were an appreciable proportion of the pipe radius $R(0.04 \lesssim \lambda / R \lesssim 0.3)$. A later study by Napoli et al. (2008) suggested that for surfaces with low effective slope, $\Delta U^{+}$scales relatively well with $E S_{x}$, irrespective of viscous scaled roughness height $k_{a}^{+}$. Again, this alludes to non $k$-type behaviour (but their low $E S$ surfaces were not tested at a range of $k^{+}$to test adherence to the fully-rough asymptote). Importantly, Napoli et al. (2008) also demonstrates that surfaces with $E S_{x}=0.05$ do not exhibit flow separation, irrespective of $k^{+}$(and blockage ratio which varied from $0.015 \lesssim k_{t} / h \lesssim 0.1$ ), while surfaces with $E S_{x}=0.15$ do. The case $c_{8}$ from Napoli et al. (2008) is especially notable in the context of the present study. This single mode two-dimensional sinusoid with $\lambda / \delta=2 \pi$ returned a lower $\Delta U^{+}$than a surface with matched $E S$ but much lower $k^{+}$, and hence much lower $\lambda / \delta$ (case $c_{1}$ ), hinting that $\lambda / \delta$ as well as low effective slope may also play a role in anomalous behaviour. Schultz \& Flack (2009) studied systematically varied threedimensional pyramid roughness, finding that for high effective slopes $\left(E S_{x}>0.35\right), \Delta U^{+}$ is more strongly dependent on the roughness height rather than $E S_{x}$, while their lowest $E S_{x}$ cases exhibited anomalous behaviour (non $k$-type behaviour), seemingly scaling with neither $k$ nor $E S_{x}$. Although the above two studies focused on low $E S_{x}$ as the cause of the anomalous results, it is noteworthy that both had large $\lambda / \delta$ ( $>1$ for certain cases in Napoli et al. (2008), and $\approx 0.3$ for non $k$-type surfaces in Schultz \& Flack (2009)).

Based on the curious findings highlighted above, and also in light of Townsend's original stipulation that the roughness length-scale must be small relative to the boundary layer thickness, we here describe a systematic study over a three-dimensional irregular surface roughness where we investigate the importance of the ratio of in-plane roughness wavelength, $\lambda$, to the boundary layer thickness $\delta$. Through uniform geometric scaling, where we scale a surface roughness by a scale factor that is the same in all directions, we vary the roughness mean amplitude $k_{a}$ and wavelength $\lambda$ while keeping the ratio $k_{a} / \lambda$ and hence $E S$ constant. By constructing surfaces with different scaling factors, and testing them in the same facility, we can vary $\delta / \lambda$ while keeping all other key parameters unchanged. We also conduct measurements at different streamwise and spanwise locations to extend the range of observable $\delta / \lambda$ while providing a measure of the degree of threedimensionality imposed on the flow by the surface roughness. 


\section{Methods}

\subsection{Facility}

For this study, the streamwise, spanwise, and wall-normal directions are represented by $x, y$, and $z$ respectively, with corresponding velocity components denoted by $u, v$, and $w$. All experiments are carried out in an open-return blower wind tunnel that consists of a settling chamber with honeycomb and five mesh screens. The tunnel has a contraction area ratio of $8.9: 1$, with a cross-sectional area of $0.94 \times 0.375 \mathrm{~m}^{2}$ and working section length of $6.7 \mathrm{~m}$. The working section floor is a steel plate that can be lowered using hydraulic lifts, allowing a seamless interchange between the smooth and rough surface. A strip of P40 sand paper immediately upstream of the inlet to the working section trips the boundary layer to turbulent.

\subsection{Surface roughness topography}

The surface roughness topography is obtained from a silicone rubber imprint acquired during dry-docking from a recently cleaned and painted ship-hull (see Hutchins et al. 2016, for further details). The resulting imprint is then scanned using a Keyence LK-031 laser triangulation sensor which has a spot-size of approximately $30 \mu \mathrm{m}$ and a vertical resolution $(z)$ of $1 \mu \mathrm{m}$. The sensor is scanned over the surface using a high precision two-axis computer controlled positioning system, with a horizontal ( $x$ and $y$ ) step-over distance of $20 \mu \mathrm{m}$. Tabulated surface properties and a height map of the resulting scanned topography are shown in figure 1. The three-dimensional rendering of the topology reveals a clear 'orange-peel' type pattern resulting from the spraying process. From the tabulated data it is noted that values of skewness and kurtosis are very close to Gaussian $\left(k_{\text {skew }} \approx 0\right.$ and $k_{\text {kurt }} \approx 3$ ). In addition, the similarity between the effective slope in the $x$ and $y$ directions $\left(E S_{x} \approx E S_{y}\right)$ indicates that the surface is nominally isotropic. The in-plane wavelength $\lambda$ of this surface is estimated at $\approx 3.4 \mathrm{~mm}$ based on the ratio of spectral moments, $\lambda /(2 \pi)=m_{0} / m_{1}$, where the $n$th spectral moment is given by $m_{n}=\int_{0}^{\infty} \kappa^{n} E_{z^{\prime} z^{\prime}} d \kappa$ and where $E_{z^{\prime} z^{\prime}}$ is the power spectral density of surface elevation, and $\mathrm{K}$ is the wavenumber $\equiv 2 \pi / \lambda$. In the shipping industry, the average peak-to-trough roughness height over a $50 \mathrm{~mm}$ fetch of the surface $\left(R t_{50}\right)$ is often used as a roughness lengthscale, which for this surface yields $R t_{50}=0.264 \mathrm{~mm}$.

\subsection{Tile manufacturing}

The topography from the surface scan is scaled and replicated to form the test surface in the wind-tunnel facility. The test surface is produced from a series of tessellating $505 \times 285 \mathrm{~mm}$ tiles manufactured using a similar method to that described in Nugroho et al. (2013). A three-axis CNC machine is used to create a master tile made of wax. The $505 \times 285 \mathrm{~mm}$ tile size is determined by the cutting dimension limit of the CNC machine, and also the required final test surface size. A platinum cured rubber mould is then formed around the master tile to create the negative roughness pattern. Finally, the negative mould is used to cast multiple polyurethane copies of the original master tile. In total, for each surface tested, we produce 30 tiles to form a rough surface over the first $5.05 \mathrm{~m}$ fetch of the working section.

Figure 2 shows relief maps of (a) the original scan, (b) a $2.5 \times$ scaled roughness tile, and (c) the $15 \times$ scaled roughness tile. The solid black line on the original roughness scan (figure 2a) illustrates the $40.4 \times 38 \mathrm{~mm}$ subset of the original scan that is used to construct the $2.5 \times$ scaled roughness tile (figure $2 \mathrm{~b}$ ). This subset is isolated from the original scan and then uniformly scaled (in all three directions) by a factor 2.5 , yielding a $101 \times 95 \mathrm{~mm}$ surface area. Periodicity is enforced via blended interpolation at the perimeter of this 


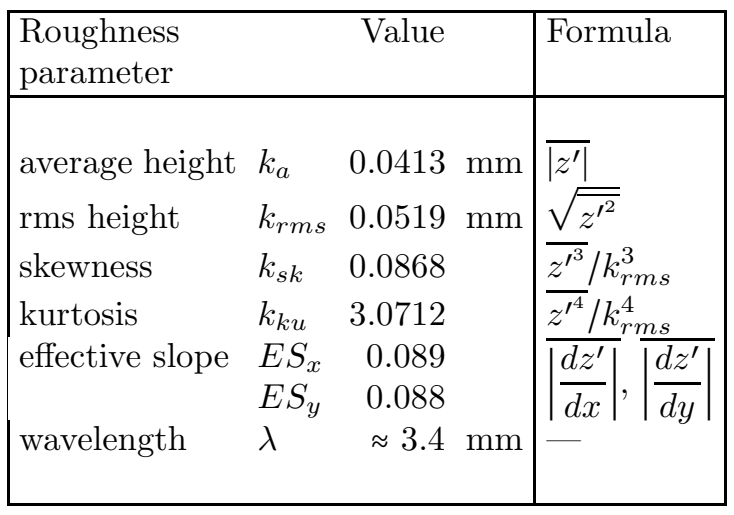

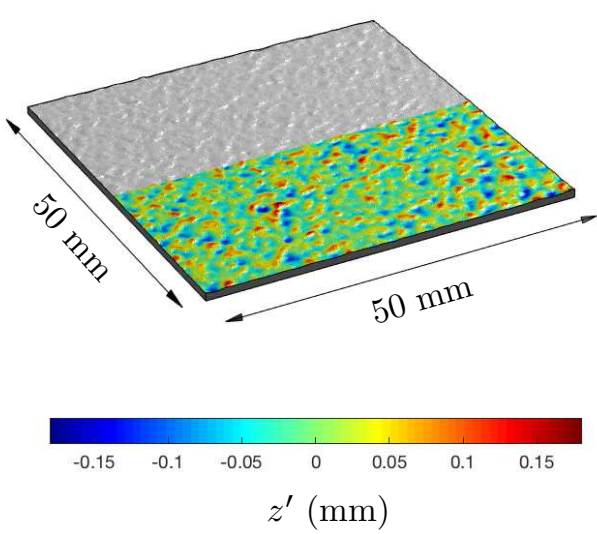

Figure 1. (left) Table of surface properties from laser scanned roughness topography and (right) three-dimensional rendering of scanned surface. Half of the surface is shown as a colour contour height map, while the other half shows the texture map.

scaled subset area, to ensure seamless tessellation. This process of enforcing periodicity effects the geometry close to the perimeter of the extracted area. However, as evident in Table 1 , the roughness parameters are only moderately effected. The $2.5 \times$ tile is formed by replicating this scaled subset five times in the $x$ direction and three times in the $y$ direction, resulting in a periodic roughness tile with the desired surface area of $505 \times 285$ $\mathrm{mm}$. The dashed black lines on the original roughness scan (figure 2a) represent the subset of the original scan extracted for the $15 \times$ scaled roughness tile (figure $2 \mathrm{c}$ ). For this scaling dimension, we isolate from the original scan an area of $33.67 \times 19 \mathrm{~mm}$ and then scale it $15 \times$ to yield the desired $505 \times 285 \mathrm{~mm}$ tile. As is clear from Table 1 , this process preserves the $k_{r m s}$ statistics from the original scan but the edge blending has resulted in an $\approx 10 \%$ reduction in $E S_{x}$ for the scaled tiles as compared to the original scanned imprint. However, both $2.5 \times$ and $15 \times$ tiles have closely matched $E S_{x}$.

The scaling in this process is typically applied to either match the expected viscous scaled conditions in the wind-tunnel to those encountered on the ship, or to ensure that the surface can be driven fully rough within the range of conditions attainable by the facility. In this case, two surfaces with distinct $2.5 \times$ and $15 \times$ uniform geometric scalings (same scaling factor applied in $x, y$ and $z$ directions) were manufactured. It is noted that for increasing geometric scaling, only the roughness height $\left(k_{a}, k_{r m s}\right)$ and the inplane roughness wavelength $(\lambda)$ will increase, whereas the effective slope $\left(E S_{x}, E S_{y}\right)$ and the ratio of height-to-wavelength (e.g. $R t_{50} / \lambda$ ) remains invariant. Typically then, under currently held ( $k$-type) assumptions, one would expect the primary outcome of the increased scaling $(15 \times)$ to be a six-fold increase in the equivalent sandgrain roughness $k_{s}$ as compared to the smaller surface $(2.5 \times)$.

\subsection{Experimental conditions}

Upper case velocity components represent time averaged or mean values and the superscript + shows viscous scaling, for example: $U^{+}=U / U_{\tau}$ for velocities and $z^{+}=z U_{\tau} / \nu$ for wall-normal distance. The boundary layer thickness $\delta$ is the wall-normal position where the mean velocity $U$ recovers to $98 \%$ of the freestream velocity $U_{\infty}$. Since the surface roughness (particularly the $15 \times$ surface) can introduce spanwise heterogeneity, we will decompose total velocities $u$ at a particular $x$ location both in terms of the 
(a) Original roughness scan

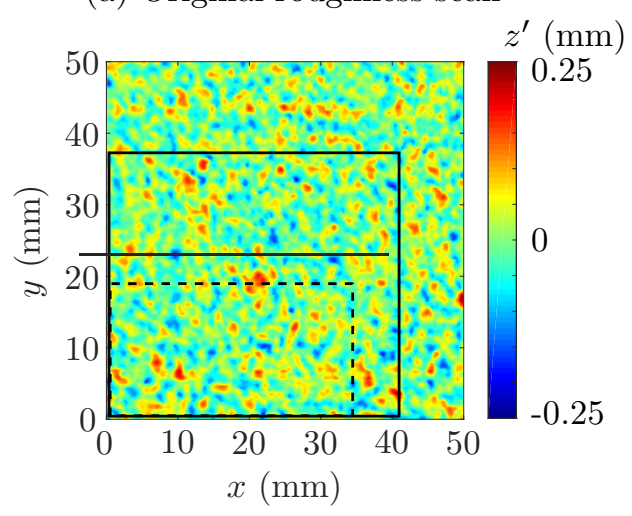

(b) $2.5 \times$ scaled roughness tile

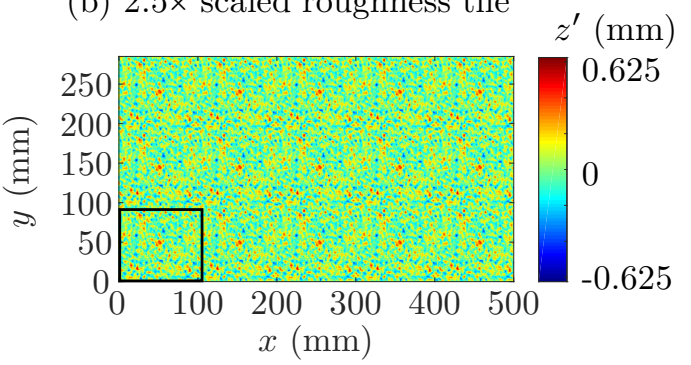

(c) $15 \times$ scaled roughness tile

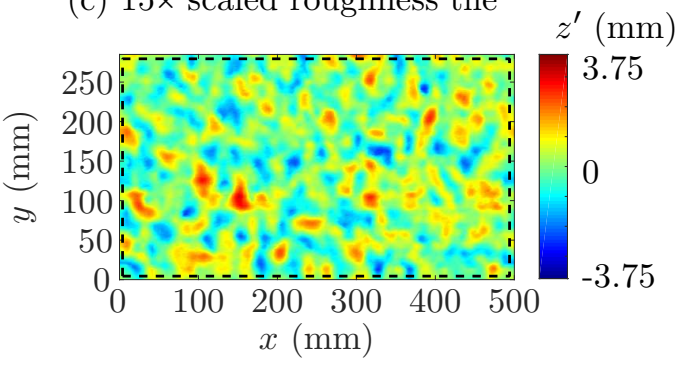

FiguRE 2. Surface roughness pattern for: (a) original roughness scan, (b) the $2.5 \times$ scaled roughness tile, and (c) the $15 \times$ scaled roughness tile. The solid black line on (a) shows the repeating subarea from the original roughness scan used to construct the $2.5 \times$ scaled roughness tile, while the dashed black line on (a) shows the subarea used to construct the $15 \times$ scaled roughness tile.

Reynolds decomposition,

$$
u=U(y, z)+u^{\prime}(y, z)
$$

and the triple decomposition (see for example Reynolds \& Hussain 1972)

$$
u=\langle U\rangle(z)+\tilde{u}(y, z)+u^{\prime}(y, z)
$$

Here $\mathrm{U}$ is the local time-averaged mean, which is a function of spanwise and wall-normal position, and $u^{\prime}$ is the fluctuation about this mean. $\langle U\rangle$ is the spatial and temporally averaged mean, which is also known as the global mean $(\langle\rangle$ denotes spanwise averaging). The coherent or dispersive component $\tilde{u}$ is the spatial variation of the time-averaged flow around individual roughness elements which is defined as $\tilde{u}=U-\langle U\rangle$. For the majority of this report we focus on the spanwise averaged mean velocity profiles $\langle U\rangle$ and the spanwise averaged turbulence variance profiles $\left\langle u^{\prime^{2}}\right\rangle$, while the coherent component $\tilde{u}$ is analysed in figure 6 .

\subsection{Flow measurement technique}

Measurements are conducted over the two scaled roughness topographies along with a corresponding smooth wall case for reference. All experiments are performed at zero pressure gradient (ZPG) conditions and at various freestream velocities $U_{\infty}$. Boundary layer profiles are measured with hot-wire anemometry (HWA) using an in-house designed 
The influence of roughness wavelength and average height on TBL

\begin{tabular}{|c|c|c|c|c|c|c|c|c|c|c|c|c|c|}
\hline Wall type & $\begin{array}{c}U_{\infty} \\
(\mathrm{m} / \mathrm{s})\end{array}$ & $\begin{array}{c}\mathrm{x} \\
(\mathrm{m})\end{array}$ & $\begin{array}{l}k_{r m s} \\
(\mathrm{~mm})\end{array}$ & $\frac{\delta}{4 k_{r m s}}$ & $E S_{x}$ & $\begin{array}{c}\lambda \\
(\mathrm{mm})\end{array}$ & $\delta / \lambda$ & $\begin{array}{c}\delta \\
(\mathrm{m})\end{array}$ & $\begin{array}{c}U_{\tau} \\
(\mathrm{m} / \mathrm{s})\end{array}$ & $R e_{\tau}$ & $\Delta U^{+}$ & $l^{+}$ & $\begin{array}{c}\text { sym- } \\
\text { bol }\end{array}$ \\
\hline smooth & $\overline{15}$ & $\overline{2}$ & - & - & - & - & 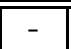 & 0.031 & 0.59 & $\mid 1200$ & - & 39 & - \\
\hline smooth & 15 & 4 & - & - & - & - & - & 0.050 & 0.55 & 1800 & - & 37 & - \\
\hline rough $2.5 \times$ & 10 & 2 & 0.13 & 67 & 0.081 & 8.5 & 4.1 & 0.035 & 0.41 & 900 & 0.83 & 27 & $\square$ \\
\hline rough $2.5 \times$ & 15 & 2 & 0.13 & 67 & 0.081 & 8.5 & 3.8 & 0.032 & 0.62 & 1300 & 1.73 & 41 & 0 \\
\hline rough $2.5 \times$ & 20 & 2 & 0.13 & 65 & 0.081 & 8.5 & 4.0 & 0.034 & 0.84 & 1800 & 2.91 & 56 & $\triangle$ \\
\hline rough $2.5 \times$ & 25 & 2 & 0.13 & 71 & 0.081 & 8.5 & 4.4 & 0.037 & 1.06 & 2500 & 3.83 & 71 & $\diamond$ \\
\hline rough $2.5 \times$ & 10 & 4 & 0.13 & 115 & 0.081 & 8.5 & 7.1 & 0.060 & 0.39 & 1500 & 0.74 & 26 & $\square$ \\
\hline rough $2.5 \times$ & 15 & 4 & 0.13 & 111 & 0.081 & 8.5 & 6.8 & 0.058 & 0.58 & 2200 & 1.36 & 39 & 0 \\
\hline rough $2.5 \times$ & 20 & 4 & 0.13 & 113 & 0.081 & 8.5 & 6.9 & 0.059 & 0.79 & 3000 & 2.22 & 52 & $\triangle$ \\
\hline rough $2.5 \times$ & 25 & 4 & 0.13 & 117 & 0.081 & 8.5 & 7.2 & 0.061 & 0.99 & 3900 & 2.83 & 66 & $\diamond$ \\
\hline rough $15 \times$ & 10 & 2 & 0.78 & 13 & \begin{tabular}{|l|}
0.079 \\
\end{tabular} & 51 & 0.8 & 0.040 & 0.46 & 1200 & 3.43 & \begin{tabular}{|l|}
30 \\
\end{tabular} & 口 \\
\hline rough $15 \times$ & 15 & 2 & 0.78 & 12 & 0.079 & 51 & 0.8 & 0.039 & 0.67 & 1700 & 4.20 & 44 & 0 \\
\hline rough $15 \times$ & 20 & 2 & 0.78 & 13 & 0.079 & 51 & 0.8 & 0.041 & 0.87 & 2400 & 4.15 & 58 & $\Delta$ \\
\hline rough $15 \times$ & 25 & 2 & 0.78 & 14 & 0.079 & 51 & 0.9 & 0.044 & 1.08 & 3200 & 4.48 & 72 & $\checkmark$ \\
\hline rough $15 \times$ & 10 & 4 & 0.78 & 22 & 0.079 & 51 & 1.4 & 0.070 & 0.41 & 1900 & 1.99 & 27 & $\square$ \\
\hline rough $15 \times$ & 15 & 4 & 0.78 & 22 & 0.079 & 51 & 1.4 & 0.069 & 0.60 & 2700 & 2.39 & 40 & 0 \\
\hline rough $15 \times$ & 20 & 4 & 0.78 & 22 & 0.079 & 51 & 1.4 & 0.070 & 0.78 & 3600 & 2.23 & 52 & $\triangle$ \\
\hline rough $15 \times$ & 25 & 4 & 0.78 & 23 & 0.079 & 51 & 1.4 & 0.071 & 0.97 & 4600 & 2.51 & 65 & $\diamond$ \\
\hline
\end{tabular}

TABLE 1. Key flow parameters

Melbourne University constant temperature anemometer (MUCTA) following the design of Perry (1982). The hot-wire probe is a single-normal type Auspex A55P05 boundary layer probe connected to a $4 \mathrm{~mm}$ diameter probe support (Dantec 55H21). The sensing element of the hot-wire is platinum Wollaston wire with $5 \mu \mathrm{m}$ diameter. To minimise attenuation due to end conduction, the sensing element is etched to length $1 \mathrm{~mm}$ resulting in a length-to-diameter ratio $l / d \approx 200$ (Ligrani \& Bradshaw 1987; Hutchins et al. 2009). Because measurements are conducted with the same sensor across a range of freestream velocities, the viscous scaled wire length $l^{+}$will vary between $\approx 25-65$. Thus at the higher speeds the measurements will increasingly suffer from attenuation due to insufficient spatial resolution (Hutchins et al. 2009). Full calibrations are conducted in the freestream next to a Pitot-static tube prior to and after each experiment. To account for drift during experiments, we follow the method of Talluru et al. (2014) where the boundary layer profile is periodically interrupted to relocate the hot-wire to the free stream to obtain a single recalibration point. The hot wire is attached to a traverse that is capable of moving in both the spanwise and wall-normal directions and for the rough wall surface we conduct a full two-dimensional flow mapping, where the hot wire is traversed over a spanwise and wall-normal plane which measures $150 \times 150 \mathrm{~mm}(\gtrsim 2 \delta \times 2 \delta$ at $x=4 \mathrm{~m})$. The plane has 11 linearly spaced points in the spanwise direction and 31 logarithmically spaced points in the wall-normal direction. To minimise drift over the duration of the two-dimensional measurements (630 individual measurement locations), total sample duration at each measurement station is reduced to $30 \mathrm{~s}$ at $50 \mathrm{kHz}$, corresponding to a boundary layer turn over time of $\approx 5000 \delta / U_{\infty}$ at $x=4 \mathrm{~m}$. The maximum viscous scaled sample interval is $\Delta t^{+}<1.3$. For the smooth wall cases, where the boundary layer can be considered spanwise homogeneous, wall-normal profiles were made at a single spanwise location corresponding to the tunnel centerline, and consisted of 50 logarithmically-spaced points with 150 s sample durations.

Measurements are made at two different streamwise locations and four different 
freestream velocities for all three surface types (smooth wall, $2.5 \times$ and $15 \times$ scaled), see table 1 for further details. The majority of the reporting here will focus on the measurements made at the downstream location $(x=4 \mathrm{~m})$. Data from the upstream location are considered in $\S 3.3$.

\subsection{Estimating skin friction}

To estimate skin friction velocity over the smooth wall reference case we implement the Clauser (1954) method by fitting the measured mean velocity to a log-law with constants $\kappa=0.39$ and smooth wall intercept $A=4.3$ (see Marusic et al. (2013) for the universality of these log law constants). In the rough wall case, where the hot wire measurements are made over a $2 \mathrm{D}$ spanwise / wall-normal plane, the friction velocity $U_{\tau}$ is obtained from the spanwise avereraged mean profiles or global mean $\langle U\rangle$. Obtaining $U_{\tau}$ for rough wall data using the Clauser method has added uncertainty owing to additional unknown fitting parameters: the Hama roughness function $\Delta U^{+}$and also the wall-normal adjustment due to the shifted aerodynamic origin $e$ of the rough surface. Though one can iteratively search the optimal combination of $U_{\tau}$ and $e$ to yield the required gradient $1 / \kappa$ in the logarithmic region, the uncertainties are rather high. Schultz \& Flack (2007) estimate the error in the modified Clauser method (following the approach of Perry \& Li (1990)) at $\pm 4 \%$ for rough walls, although this error would be expected to increase where the extent of the logarithmic region is curtailed, as occurs in experiments with low Reynolds numbers, high $k / \delta$, or a thick roughness sublayer. Due to a combination of these factors in the present study, we follow the approach that was proposed by Monty et al. (2011), where Townsend's (1976) outer layer similarity is assumed to hold in both the mean velocity and variance profiles. The correct value for $U_{\tau}$ is then assumed to be that which best collapses both the mean velocity defect and turbulence intensity data (scaled with $U_{\tau}$ and $\delta$ ) in the outer region of the flow $(0.2 \delta<z<\delta)$. This method is perhaps advantageous in situations where the extent of the log region may be limited. The values of $U_{\tau}$ obtained from the modified Clauser method and the assumed outer layer similarity method (Monty et al. 2011) agree with each other to within $9 \%$. In this instance the method of Monty et al. (2011) is preferred due to the aforementioned limitations in making the Clauser estimate. However, it is noted that the major conclusions from this study hold regardless of which estimate for $U_{\tau}$ is taken. Indeed, in light of the surprising results from this study, the momentum deficit has also been used to obtain a further (and more direct) estimate of $U_{\tau}$. The momentum thickness at the streamwise measurement station provides a measure of the integrated skin friction coefficient (and hence integrated $U_{\tau}$ ) along the test plate. In this case, the momentum thickness variation observed between the cases detailed in table 1 exhibits exactly the same surprising trends as those indicated by $U_{\tau}$ as calculated via Clauser or assumed outer layer similarity methods. In short, the observed trends and major conclusions from this study in no way depend on the choice of estimate for $U_{\tau}$, with all estimates pointing to the same (surprising) behaviour.

\section{Results}

\subsection{Scaling and turbulence statistics}

Figure 3 shows the time-averaged statistics for the $2.5 \times$ scaled roughness at the streamwise location $x=4 \mathrm{~m}$ (open symbols as defined in table 1) along with the corresponding smooth wall reference case (solid lines). Figure 3(a) shows the innernormalised mean velocity profiles showing the downward shift $\Delta U^{+}$for the rough surface relative to the smooth wall case. Note that spanwise averaged mean profiles are plotted 
(a)

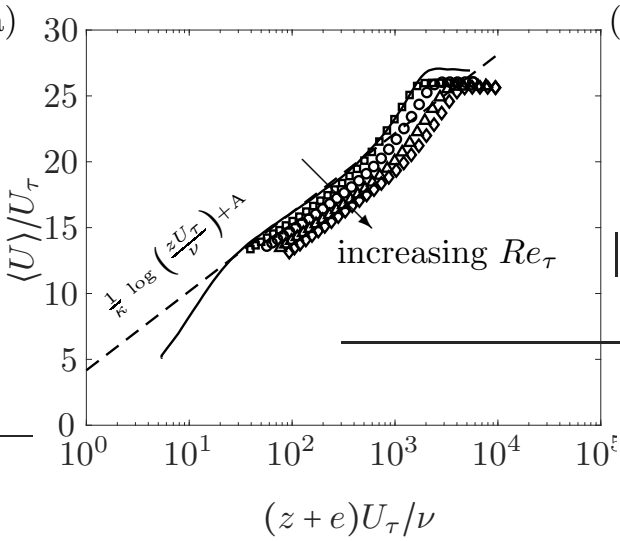

(b)

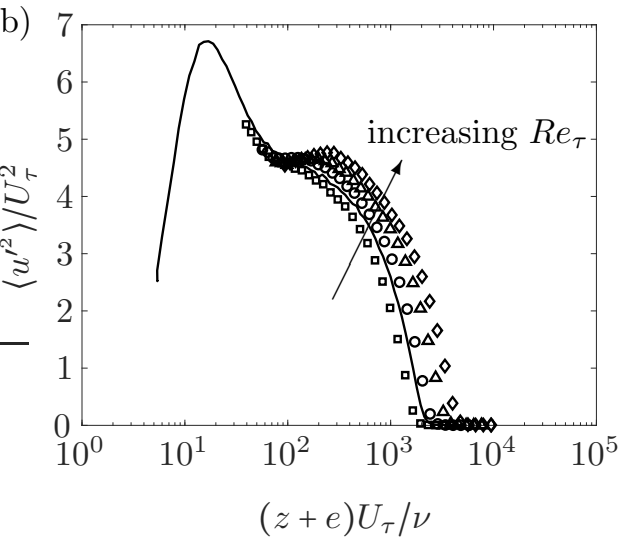

(d)

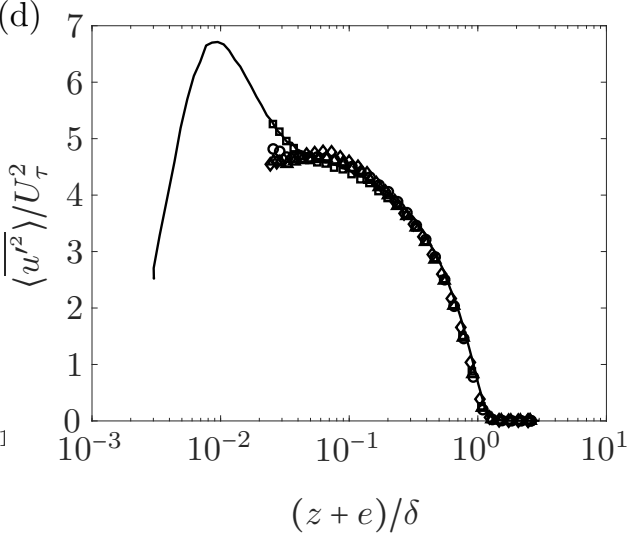

Figure 3. Mean profiles from the $2.5 \times$ rough surface at $x=4 \mathrm{~m}$ (a) inner-scaled mean velocity profile; (b) inner-scaled turbulence intensity; (c) mean velocity defect; (d) outer-scaled turbulence intensity. Symbols are as defined in table 1. Solid line shows reference smooth wall data at $x=4 \mathrm{~m}$ and $U_{\infty}=15 \mathrm{~ms}^{-1}$.

in this figure, as indicated by the angled brackets. The downward shift in $\langle U\rangle^{+}$increases with increasing freestream velocity as the roughness Reynolds number $\operatorname{Re}_{k}\left(\equiv k_{a} U_{\infty} / \nu\right)$ also increases. Figure 3(b) plots the inner scaled spanwise averaged turbulence intensity of the streamwise velocity fluctuations $\left\langle\overline{u^{\prime 2}}\right\rangle^{+}$. Note that these are fluctuations about the local time-averaged mean and do not include the dispersive stress $\tilde{u}$ (see equations 2.1 and 2.2). Here the smooth wall case exhibits a near-wall peak at $z^{+}=15$, signalling the highly energetic near-wall cycle of streaks and quasi streamwise vortices (Kline et al. 1967). Unfortunately, for the rough wall case we are unable to reach $z^{+}=15$ due to the physical constraint of the hot wire size (the probe was blocked by the surrounding roughness peaks). Figures $3(\mathrm{c})$ and (d) show the mean streamwise velocity defect profile and outer scaled streamwise turbulence intensity respectively. Both figures clearly show the simultaneous collapse of rough wall flow statistics on to the smooth wall reference cases. Though the method of Monty et al. (2011) assumes outer layer similarity to find $U_{\tau}$, there is no a priori requirement for simultaneous collapse in both the defect and variance profiles as is observed in figures $3(\mathrm{c})$ and $(\mathrm{d})$.

Figure 4 shows the mean profiles for the $15 \times$ scaled roughness. Figure $4(\mathrm{a})$ shows the inner-scaled mean velocity profiles. Here, unlike the $2.5 \times$ scaled roughness case, the mean velocity profiles for the $15 \times$ scaled roughness do not behave as we would expect for a traditional ( $k$-type) rough wall flow. As $U_{\infty}$ (and roughness Reynolds number) 

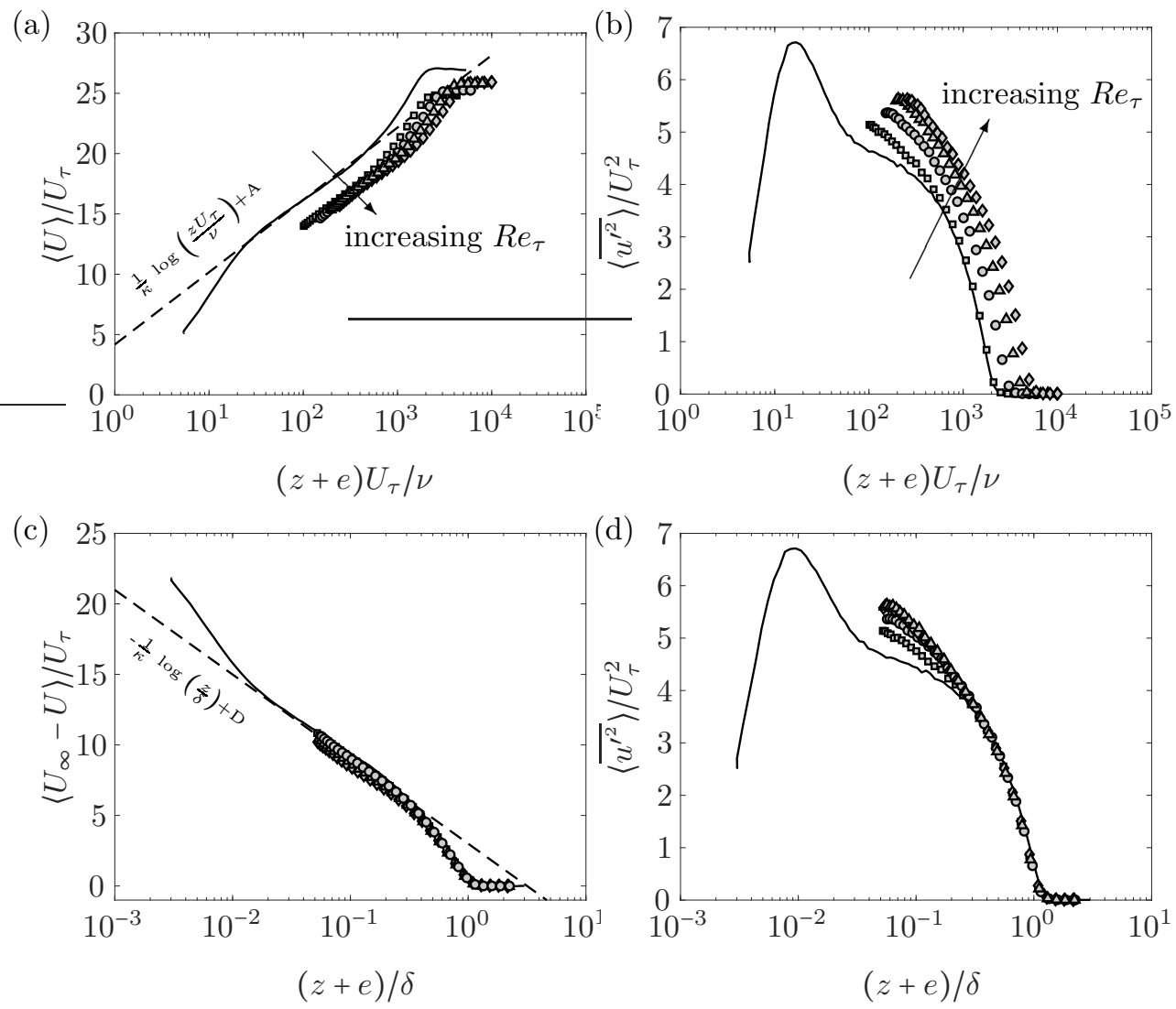

FiguRE 4. Mean profiles from the $15 \times$ rough surface at $x=4 \mathrm{~m}$ (a) inner-scaled mean velocity profile; (b) inner-scaled turbulence intensity; (c) mean velocity defect; (d) outer-scaled turbulence intensity. Symbols are as defined in table 1. Solid line shows reference smooth wall data at $x=4 \mathrm{~m}$ and $U_{\infty}=15 \mathrm{~ms}^{-1}$.

increases, there is only a very marginal increase in the downward shift $\Delta U^{+}$exhibited by the profiles. This relatively constant shift is puzzling. Between the highest and lowest freestream velocity cases shown in figure 4(a), the friction velocity (and hence the viscous scaled roughness height) has more than doubled, for which we would typically expect a wide variation in $\Delta U^{+}$. Indeed when we compare the viscous-scaled mean velocity profiles for the $2.5 \times$ and the $15 \times$ surfaces (figures $3 \mathrm{a}$ and $4 \mathrm{a}$ ), despite the six-fold increase in roughness height between the two cases, the $\Delta U^{+}$has remained comparable. Despite this anomalous behaviour, the velocity defect profile of figure 4(c) and outer scaled streamwise turbulence intensity of figure $4(\mathrm{~d})$ exhibit a similar approximate collapse to smooth wall profiles, as observed previously for the $2.5 \times$ data. The only subtle difference here is that outer layer similarity in the variance profile is restricted to a region further from the wall $(z / \delta \gtrsim 0.4)$ for the $15 \times$ scaled surface. Presumably this is related to dispersive stresses / secondary flows that extend much further from the surface for the $15 \times$ case (see §3.2.1). Indeed, Chan et al. (2018) suggest that the secondary flows (and hence the roughness sublayer) extend up to $z=0.5 \lambda$, which equates to $z / \delta \approx 0.4$ for the $15 \times$ case. This corresponds closely to the point beyond which outer layer similarity is observed in the variance profiles of figure $4(d)$.

To better highlight the surprising behaviour of the $15 \times$ surface, figure 5 plots the Hama roughness function $\Delta U^{+}$as a function of the viscous scaled average roughness 


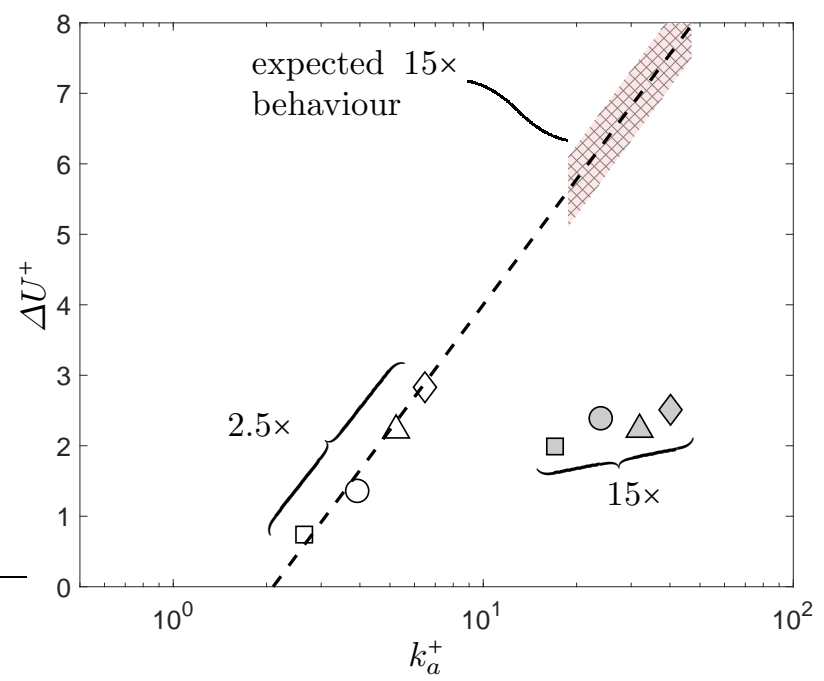

FigURE 5. Hama roughness function $\Delta U^{+}$against the viscous scaled mean roghness height $k_{a}^{+}$ at $x=4 \mathrm{~m}$ for the (open symbols) $2.5 \times$ and (filled) $15 \times$ surfaces. Symbols are as defined in table 1. Dashed line shows fully rough asymptote fitted to the $2.5 \times$ data, $\Delta U^{+}=\kappa^{-1} \ln \left(C k_{a}^{+}\right)+A-B$, where the constant $C \approx 2.45$ as determined by least-squares fitting. Hatched region shows the expected behaviour for the $15 \times$ surface based on the $2.5 \times$ result and an assumed $k$-type behaviour (using the predictive method of Monty et al. (2016)).

height $k_{a}^{+}$. The two different scalings exhibit very different behaviour. The $2.5 \times$ data shows a downward shift $\Delta U^{+}$that increases with roughness Reynolds number, seeming to follow the expected log-linear behaviour for a fully rough $k$-type flow,

$$
\Delta U^{+}=\frac{1}{\kappa} \ln C k_{a}^{+}+A-B
$$

where $A$ is the smooth wall $\log$ law intercept and $B=8.5$ is Nikuradse's (1933) fully rough constant. Here $C$ is a scaling factor that links $k_{a}$ to $k_{s}$. For the $2.5 \times$ data a value $C \approx 2.45$ yields a good fit to the above trend, suggesting that the equivalent sand grain roughness $k_{s}$ for the $2.5 \times$ surface is $0.253 \mathrm{~mm}$. The behaviour of the $15 \times$ scaled surface is very different. This surface has a $k_{a}$ value that is six times greater than for the $2.5 \times$ surface, with the same solidity or effective slope, and thus we would expect very large values of $\Delta U^{+}$for this surface. As an approximate rule of thumb, since $C$ would be expected to remain invariant for a geometrically scaled surface, the range of freestream velocities $10 \leq U_{\infty} \leq 25 \mathrm{~ms}^{-1}$ would be expected to yield a Hama roughness function for the $15 \times$ surface in the range $5.6<\Delta U^{+}<8.5$, as based on the $2.5 \times$ results (equation 3.1) and the predictive method outlined in Monty et al. (2016). However, figure 5 shows unambiguously that this is not the case. The expected range of results (based on assumed $k$-type behaviour) is shown by the hatched region. The measured results show that, despite the six-fold increase in roughness height, the magnitude of the Hama roughness function has barely changed between the $15 \times$ case as compared to the $2.5 \times$. Moreover, the trend of $\Delta U^{+}$against $k_{a}^{+}$for the $15 \times$ surface shows no signs of approaching the expected fully rough asymptotic behaviour as given by equation (3.1). From this figure it can be concluded that the $2.5 \times$ surface behaves as a typical $k$-type roughness, while the $15 \times$ surface resolutely does not. 

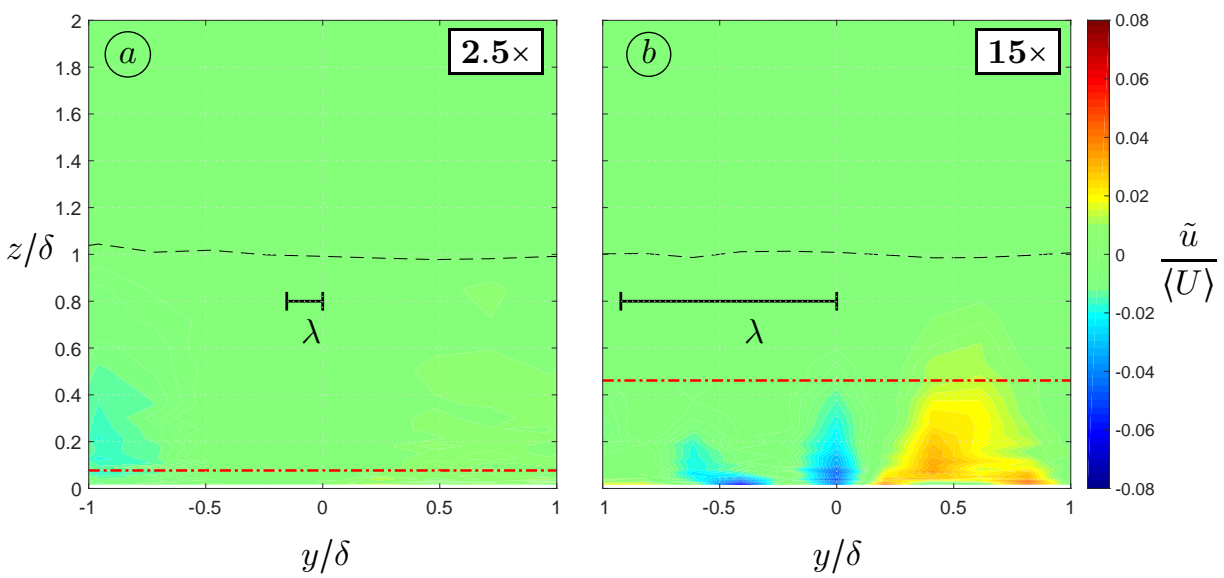

FiguRE 6 . The spanwise variation in the coherent or dispersive streamwise velocity component $\tilde{u}$ as defined in equation (2.2). Shaded contours show the variation in the local coherent component normalised by the global mean $\tilde{u} /\langle U\rangle \equiv(U-\langle U\rangle) /\langle U\rangle$. for the $(a) 2.5 \times$ and $(b) 15 \times$ surfaces. The dot-dashed line shows the approximate limit of the dispersive stresses $z=0.5 \lambda_{y}$ as given by Chan et al. (2018). Dashed lines show spanwise variation in the boundary layer thickness $\delta$ and the in-plane roughness wavelength $\lambda$ is represented by the included measurement scale.

The topographical properties collated in table 1 show that the only changes that can have given rise to this anomalous behaviour are either the reduction in the ratio of boundary layer thickness to in-plane roughness wavelength $\delta / \lambda$ or the increasing blockage effect as $\delta / 4 k_{r m s}$ reduces. We choose the measure $4 k_{r m s}$ in this instance for the characteristic roughness height based on the observation that the surface closely resembles a three-dimensional sinusoidal surface, which would have a peak-to-trough roughness height of $4 k_{r m s}$. In this case, it is unlikely that blockage for the $15 \times$ case would be sufficient to cause this non $k$-type behaviour. The ratios of $\delta / 4 k_{r m s}$ listed in table 1 at $x=4 \mathrm{~m}$ are approximately half of the 40 suggested by Jiménez (2004). Indeed, there have been many studies that have substantially relaxed the ratio $\delta / k=40$ to values that are comparable to, or lower than, those listed in table 1 for the $15 \times$ case, while still observing $k$-type behaviour and outer layer similarity (Flack et al. 2007; Chan et al. 2015; Forooghi et al. 2017, 2018; Flack et al. 2020; Jelly \& Busse 2019, to name but a few). Townsend's assumptions for outer-layer similarity require that no roughness length-scale is competing for dominance with $\delta$ in the outer part of the flow. Typically we take $k$ as the roughness lengthscale, which may be acceptable for surfaces with higher $E S$ where $k / \lambda \sim \mathrm{O}(1)$. However, for the low effective slope surface tested here, the scaling factor of $15 \times$ produces a $\lambda$ that is becoming equivalent to $\delta$ while $k \ll \delta$.

\subsection{Why is the $15 \times$ not 'rougher'?}

We believe that the anomalous behaviour of the $15 \times$ surface is related to the fact that the in-plane roughness wavelength $\lambda$ is approaching the layer thickness $\delta$ for this case. Aside from implications of this to Townsend's (1976) outer layer similarity hypothesis as discussed above, this can also cause a strengthening of secondary flows (§3.2.1) and the possible onset of a waviness regime $(\S 3.2 .2)$. 


\subsubsection{Secondary flows}

As the in-plane roughness wavelength becomes large relative to the layer thickness $(\lambda / \delta \rightarrow 1)$, the surface will appear more and more heterogeneous to the flow. In effect the outer flow no longer sees the roughness purely in terms of a modified homogeneous boundary condition (a modifed $U_{\tau}$ ), as is the underlying assumption for Townsend's outer layer similarity argument, but rather will encounter the roughness as a spatially varying $U_{\tau}$ or heterogeneity. It has been well documented in the literature (Hinze 1967; Barros \& Christensen 2014; Anderson et al. 2015; Willingham et al. 2014, for example) that spanwise roughness heterogeneity can lead to the formation of Prandtl's secondary flows of the second kind (Bradshaw 1987), with recent evidence suggesting that the strength of these secondary flows is a strong function of the outer-scaled heterogeneity wavelength $\lambda_{h} / \delta$, reaching a maximum strength for $\lambda_{h} / \delta \approx 2$ (Chung et al. 2018; Medjnoun et al. 2018; Yang \& Anderson 2018; Anderson et al. 2018; Wangsawijaya et al. 2020). These same studies also show that the size of the secondary flows is dependant on the spanwise lengthscale $\lambda_{h}$, with finer spanwise spacing confining the secondary flows and subsequent spanwise variations to the region closer to the wall, while when $\lambda_{h} / \delta \rightarrow 2$ the secondary flows become space filling and the spanwise variations in the mean extend throughout the majority of the boundary layer. A recent numerical study by Chan et al. (2018) of homogeneous roughness has shown that the wall-normal extent of the dispersive stresses (the extent of the spanwise three-dimensionality induced by the secondary flows) is a direct function of the spanwise wavelength (for an egg-carton type three-dimensional roughness). This study shows that the thickness of the roughness sublayer (defined as the region where the flow from individual roughness elements extends) is a direct function of spanwise in-plane roughness wavelength. When this wavelength approaches $\delta$, the roughness sublayer can start to fill the layer. As evidence of this behaviour, figure $6 a$ and $b$ plots the fractional variation in the local mean $U(y, z)$ about the spanwise average mean $\langle U\rangle(z)$ for both the $2.5 \times$ and $15 \times$ surfaces respectively (this is the dispersive component $\tilde{u}$ as a fraction of $\langle U\rangle(z)$ ). It is clear that the fractional variation across the span is much more pronounced for the $15 \times$ surface, with stronger fluctuations extending further from the wall. From their analysis of egg-carton type regular rough surfaces, Chan et al. (2018) suggest that the dispersive stresses will extend to approximately $z=0.5 \lambda_{y}$ which is shown by the dot-dashed line on figure 6 . It is clear that this limit approximately describes the wall-normal extent of the strong spanwise variations for the $15 \times$ case. For reference the mean wavelength $\lambda$ of the two scaled surfaces is also represented on the plots, and it can be seen that the average spanwise lengthscale of the mean velocity variations in figure $6(b)$ is close to this wavelength. The boundary layer thickness $\delta$ is shown by the dashed line, which shows little difference in spanwise variation for either case, in keeping with the observation that the roughness sublayer only extends to approximately half of the boundary layer thickness for the $15 \times$ case, and thus has not strongly influenced the overall layer thickness. Morgan \& McKeon (2018) studied wall-bounded turbulence over a singly-periodic sinusoidal roughness with in-plane wavelengths on the order of the boundary layer thickness, noting that the roughness sublayer extended far into the wake region $(z / \delta \approx 0.6)$.

\subsubsection{The onset of the 'wavy' regime}

Figure 7(a) and (b) show a schematic of boundary layers developing over rough surfaces with the same effective slope $E S_{x}$ (or equal solidity) but with very different $\lambda / \delta$. Note that for clarity, the sketched effective slope in the schematic $(E S \approx 0.2)$ is larger than the one tested in this study $(E S \approx 0.08)$. Figure $7(a)$ shows a case where $\lambda \ll \delta$, for which case the local modifications to the flow due to the surface undulations might be expected 


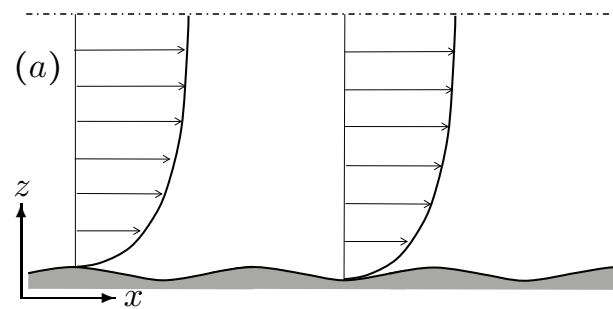

(b)

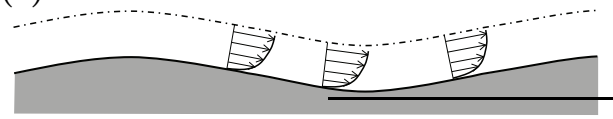

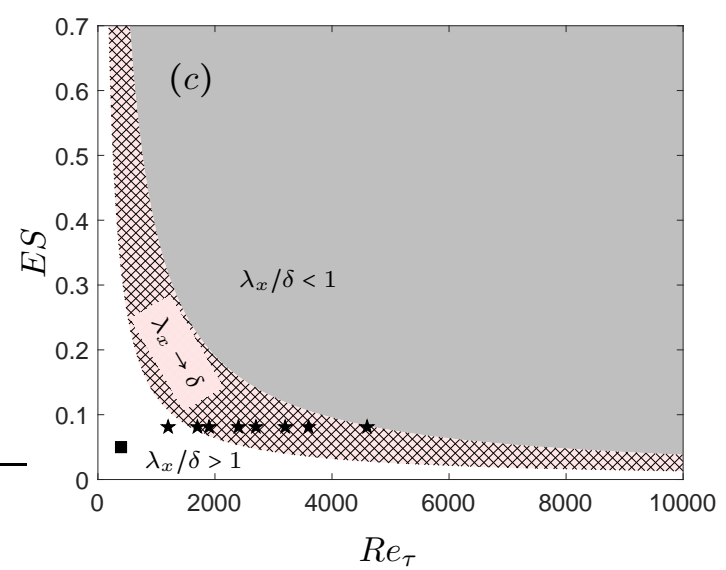

Figure 7. Two scenarios with same effective slope ES, but with $(a) \lambda_{x} / \delta<1,(b) \lambda_{x} / \delta>>1$ (c) the curve for critical $E S(\lambda \approx \delta)$ as a function of $R e_{\tau}$, for a sinusoidal roughness with $k_{p}^{+} \mathrm{O}(100)$. Symbols show conditions for the $(\star) 15 \times$ surface from the current study at both $x$ locations; (घ) two-dimensional sinusoidal surfaces of Napoli et al. (2008).

to be restricted to the near-wall region, with the average boundary layer thickness (as represented by the dot-dashed line) invariant in $x$. This is the standard homogeneous $k$ type roughness scenario that is represented by the $2.5 \times$ surface. Figure $7(b)$ shows a case where $\lambda \gg \delta$. In this limiting case, provided that the effective slope of the surface was sufficiently low such that flow separations were minimised, we might expect a scenario as shown, where the entire boundary layer locally conforms to the surface. In this case the surface undulations no longer appear to the near-wall flow as roughness (there is no roughness generated turbulence close to the surface). In such scenarios we might expect non $k$-type behaviour, with the drag being purely driven (in the limit) by the changes in surface area and by the resultant secondary flows.

An approach to the fully rough asymptote ( $k$-type behaviour) in the Hama roughness function plots of figures 5 and 8 , is typically associated with the dominance of pressure drag over viscous drag. Hence, the different roughness function curves for the two scaled surfaces may suggest that the $2.5 \times$ surface is more prone to separation and pressure drag. Conversely, the distinct Reynolds number dependence in $C_{f}$ indicated by the roughness function trend for the $15 \times$ surface suggests that viscous drag should be more dominant in this case. Certainly it is conceivable that for very low ES cases the waviness of the surface could become so gradual that viscous effects might still be important regardless of the roughness Reynolds number $k^{+}$. The suggestion from the comparison of the $2.5 \times$ and $15 \times$ surfaces is that for surfaces with matched $E S$ this dominance of viscous effects might be stronger and the pressure drag effects (and separation) weaker, in cases where $\lambda \rightarrow \delta$. It is useful to refer back to the literature at this point. Napoli et al. (2008), Nakato et al. (1985) and Schultz \& Flack (2009) have all suggested that surfaces with low effective slopes can exhibit non $k$-type behaviours, with the former two suggesting that the demarcation could lie somewhere around $E S_{x} \approx 0.15$. Indeed Napoli et al. (2008) found that flow remained attached around roughness elements for $E S_{x} \approx 0.05$, but separated for $E S_{x} \approx 0.15$. The current $2.5 \times$ and $15 \times$ scaled surfaces both have equal $E S_{x} \approx 0.08$, lying between these these two regimes. One curious aspect of the current result is that the $2.5 \times$ surface exhibits $k$-type behaviour, while the $15 \times$ surface does not, 
despite both having the same $E S_{x}$. This perhaps suggests that it is not only $E S_{x}$ that determines the predominance of pressure drag and separation from roughness elements. Along similar lines, Zenklusen et al. (2012) found that whether the flow remained attached or separated from a surface with fixed $E S_{x}=0.2$ was a function of blockage $k_{t} / H$ or $\lambda / \delta$, with attached flow occurring when $k_{t} / H=1 / 3$ and $\lambda / \delta=3$. The blockage ratio for the $15 \times$ surface tested here is much smaller $\left(k_{t} / \delta \approx 20\right)$ which perhaps suggests that $\lambda / \delta$ may be the more important determinant of this behaviour.

The scenario shown in figure $7(b)$ is certainly more extreme than the $15 \times$ surface (where $\lambda \approx \delta$ ), but this conceptual sketch does provide some insight into how surfaces with the same $E S_{x}$, but different $\lambda / \delta$ might interact with external boundary layers in very different ways. For the future, in light of the unusual Hama roughness function behaviour for the $15 \times$ surface, it would be beneficial to compare the separation or pressure drag in the roughness canopy for these surfaces. Laser doppler anemometry (LDA) or index matched particle image velocimetry (PIV) are both candidate measurement techniques that offer access to the roughness canopy and can cope with flow reversals.

If we accept that there is a demarcation between $k$-type and non $k$-type (or wavy) behaviour that occurs where $\lambda$ approaches $\delta$, we can perform simple rule-of-thumb calculations to uncover when such scenarios are likely to occur. If we assume a threedimensional sinusoidal surface (of the type studied by Chan et al. 2015) we can express the effective slope in the $x$ direction as,

$$
E S_{x}=\frac{8 k_{p}^{+}}{\pi \lambda_{x}^{+}}
$$

where $k_{p}^{+}$is the half amplitude of the sinusoidal roughness and $\lambda_{x}$ is the streamwise inplane roughness wavelength. If we assume that $\lambda_{x} / \delta=1$ at the approximate demarcation between $k$-type and non $k$-type behaviour, we can write that $\lambda_{x}^{+}=\delta^{+}=R e_{\tau}$. For most experiments aiming at fully rough conditions, $k_{p}^{+}$would be order 100 . Hence the critical effective slope $E S_{x_{c r i t}}\left(\right.$ when $\lambda_{x}=\delta$ ) can be expressed as,

$$
E S_{x_{c r i t}}=\frac{8 k_{p}^{+}}{\pi R e_{\tau}} \approx \frac{255}{R e_{\tau}}
$$

Though this is for a single mode three-dimensional sinusoidal surface, and though the actual value of $k_{p}^{+}$is unknown, (3.3) can show some important properties (if we accept that $\lambda \rightarrow \delta$ demarcates roughness regimes). Equation (3.3) is plotted in figure $7(c)$. Since the demarcation $\lambda \rightarrow \delta$ is indistinct, due to the need to assume a value for $k_{p}^{+}$, we represent the proposed transition between roughness regimes with a hatched region representing $50<k_{p}^{+}<150$. The grey shaded region in figure $7(c)$ shows the region of the parameter space where $\lambda<\delta$, while the white shaded region shows $\lambda>\delta$. Figure $7(c)$ demonstrates that for high Reynolds numbers, we would in general require abnormally low $E S$ to see $\lambda_{x} / \delta$ approach unity (under the present assumptions for the sinusoidal surface, $E S_{x_{\text {crit }}} \approx 0.025$ at $\left.R e_{\tau}=10000\right)$. However, at lower $R e_{\tau}$, more realistic $E S_{x}$ will meet this waviness criteria as fully rough conditions are approached $\left(E S_{x_{\text {crit }}}=0.255\right.$ at $\left.R e_{\tau}=1000\right)$. Certainly, for the present $15 \times$ data, it is the combination of low $E S_{x}$, relatively large roughness height $k_{p}$, and low to moderate $R e$ that have caused $\lambda / \delta$ to approach unity. Conditions from the present study for the $15 \times$ surface at $x=2 \mathrm{~m}$ and $x=4 \mathrm{~m}$ are included in figure $7(c)$ as the filled star symbols. It is clear that these data encroach on the region of the $R e_{\tau}$ and $E S$ parameter space where we expect $\lambda \approx \delta$ (the $15 \times$ surface has nominal $\lambda / \delta=1.4$ at $x=2 \mathrm{~m}$ and $\lambda / \delta=0.8$ at $x=4 \mathrm{~m}$ ). The filled square symbol in figure $7(c)$ is case $c_{8}$ from Napoli et al. (2008), which is a single mode two-dimensional sinusoid with $\lambda / \delta=2 \pi$. Napoli et al. (2008) reported a lower $\Delta U^{+}$for 


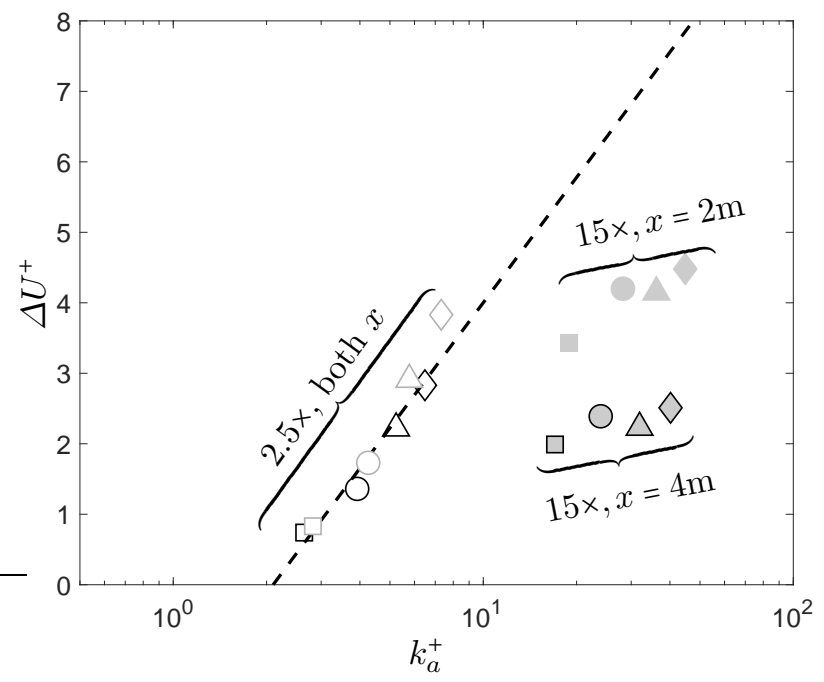

FIgURE 8. Hama roughness function $\Delta U^{+}$against the viscous scaled mean roghness height $k_{a}^{+}$ for the (open symbols) $2.5 \times$ and (filled) $15 \times$ surfaces for both the $x=4 \mathrm{~m}$ (black symbol outline) and $x=2 \mathrm{~m}$ (grey symbol outline). Symbols are as defined in table 1. Dashed line shows fully rough asymptote fitted to the $2.5 \times$ data, $\Delta U^{+}=\kappa^{-1} \ln \left(C k_{a}^{+}\right)+A-B$, where the constant $C \approx 2.45$ as determine by least-squares fitting.

this case than a surface with the same $E S$ but much lower $k^{+}$(case $c_{1}$ ). It should be reiterated that the $\lambda \approx \delta$ curves plotted on figure $7(c)$ assume a single mode egg-carton type sinusoidal surface with $k^{+} \mathrm{O}(100)$. The surfaces and conditions of Napoli et al. (2008) do not conform to these idealised assumptions. Nonetheless, the parameter space map of figure $7(c)$ may be a useful tool for collating rough surfaces that exhibit non-classical behaviour. An alternative form of $(3.3)$ is that $E S_{x_{c r i t}}=(8 / \pi)\left(k_{p} / \delta\right)$, which indicates that the critical $E S_{x}$ is only likely to be abnormally low in situations where $k_{p} / \delta$ is very small (a scenario that usually necessitates high $R e$ to achieve fully rough conditions). For more typical values of $k_{p} / \delta$ (say $0.025-0.05$, typical of many laboratory scale facilities), $\lambda / \delta$ may approach unity at much higher values of $E S_{x}$.

\subsection{A possible d-type behaviour}

Figure 8 shows the Hama roughness function for both the $x=2 \mathrm{~m}$ and $x=4 \mathrm{~m}$ measurements. Considering first the $2.5 \times$ results, it is clear that this smaller scaled surface behaves in a $k$-type manner. The values of $\Delta U^{+}$and $k_{a}^{+}$are slightly higher at matched $U_{\infty}$ for the $x=2 \mathrm{~m}$ data, as compared to the $x=4 \mathrm{~m}$ cases, however this is simply due to the variation of $C_{f}$ with $x$ for a developing turbulent boundary layer (see for example Monty et al. 2016). The important point for the $2.5 \times$ case is that when plotted in this manner, the variation of $\Delta U^{+}$with $k_{a}^{+}$for both streamwise locations conforms to the same $k$-type fully rough asymptote. The $15 \times$ data shows a more surprising tendency. In some respects the $x=2 \mathrm{~m}$ data confirms the previously observed behaviour for the $15 \times$ surface at $x=4 \mathrm{~m}$. The growth of the roughness function with $k_{a}^{+}$no longer seems to be tending to the fully rough assymptote and also, despite the six-fold increase in $k_{a}^{+}$, the Hama roughness function for the $15 \times$ surface is no larger than for the $2.5 \times$ surface. The added complication in the behaviour is that in general the $\Delta U^{+}$for the $x=2 \mathrm{~m}$ data are higher than the $x=4 \mathrm{~m}$ data, despite the fact that viscous scaled roughness height $k_{a}^{+}$is 
approximately comparable between cases. This again is strongly indicative of non $k$-type behaviour. For the $15 \times$ surface, $\lambda / \delta \approx 0.8$ for the $x=4 \mathrm{~m}$ measurements, rising to $\lambda / \delta \approx 1.4$ for the $x=2 \mathrm{~m}$ cases. The difference in $\Delta U^{+}$at these different streamwise locations (with very different boundary layer thicknesses) suggests that as $\lambda / \delta$ approaches unity, it is the ratio $\lambda / \delta$ and not the viscous scaled roughness height $k^{+}$that determines the drag penalty $\Delta U^{+}$. In this sense the comparison shown in figure 8 is suggestive of the $d$-type regime suggested by Perry and coworkers (Perry et al. 1969).

\section{Conclusions}

From these results, it is clear that although we keep $E S_{x}$ constant, geometrically scaling a roughness to obtain larger $k_{a}$ does not necessarily lead to a surface that is dynamically 'rougher'. The subtlety here is that when we scale surfaces geometrically in all dimensions, in addition to making $k$ larger, the in-plane wavelength of the roughness $\lambda$ relative to the turbulent boundary layer thickness $\delta$ is also increased. It would appear that when this wavelength becomes an appreciable proportion of the boundary layer thickness, the surface ceases to behave in a 'k-type' manner. As $\lambda$ approaches $\delta$, the roughness function (and hence drag penalty) ceases to scale with $k$, showing signs instead of scaling with $\lambda / \delta$. We show that in this condition, large-scale secondary flows (or regions of dispersive stress) form, which in the $15 \times$ case permeate the lower half of the boundary layer possibly affecting the dynamics in the logarithmic region. Ultimately, one might imagine that as $\lambda$ becomes much greater than the boundary layer thickness, the flow may experience the roughness in a very different manner as the entire boundary layer starts to 'ride over' the undulations (as represented schematically in figure $7 a$ ). In the future, a more systematic investigation that involves several additional $\lambda / \delta$ will be required to provide a better understanding of this behaviour. In addition, based on observations from the $\Delta U^{+}$curves, which suggest that the $15 \times$ case is less dominated by pressure drag, it would be useful to document any changes in flow separation about the roughness elements that might occur as $\lambda / \delta \rightarrow 1$.

Ultimately, the critical behaviour demonstrated here as $\lambda / \delta$ approaches unity suggests caution should be exercised when attempting to replicate the viscous scaled roughness condition from a large (high $R e$ ) engineering application in a much lower $R e$ laboratory facility or numerical simulation (particularly where the facility has a very different unit Reynolds number $U_{\infty} / \nu$ ). As an example, the $15 \times$ scaled surface that led to the anomalous behaviour reported here resulted from an attempt to scale a scanned ship hull roughness (resulting from spray painting application) to attain fully rough behaviour in a small-scale wind-tunnel. Typically, we scale surfaces because we wish to match viscous scaled roughness heights obtained in the laboratory to those encountered in application. However, because the $R e_{\tau}$ in the laboratory (or simulation) is often so much lower than the application Reynolds number, there is a danger for low effective slope surfaces that $\lambda$ will approach the outer lengthscale $\delta$. Results presented in this study suggest that drag predictions will suffer when this condition is artificially imposed through scaling. There are, however, likely to be other cases in engineering and meteorological applications where $\lambda$ is equivalent to the outer length scale. The stable boundary layer over long wavelength ocean waves provides one possible example, as do thin boundary layers developing over low effective slope engineering surfaces (resulting, say, from spray coating or manufacturing processes), such as narrow internal cooling channels in heat exchangers. 


\section{Declaration of Interests}

The authors report no conflict of interest.

\section{Acknowledgements}

The authors would like to thank the Australian Research Council and the Newton Fund for the support of this work. B. Ganapathisubramani is grateful to EPSRC for their support through grant EP/P009638/1.

\section{REFERENCES}

Anderson, W., Barros, J. M., Christensen, K. T. \& Awasthi, A. 2015 Numerical and experimental study of mechanisms responsible for turbulent secondary flows in boundary layer flows over spanwise heterogeneous roughness. J. Fluid Mech. 768, 316-347.

Anderson, W., Yang, J., Shrestha, K. \& Awasthi, A. 2018 Turbulent secondary flows in wall turbulence: vortex forcing, scaling arguments, and similarity solution. Environ. Fluid Mech 18, 1351-1378.

Angelis, V D, Lombardi, P \& BAnerJee, S 1997 Direct numerical simulation of turbulent flow over a wavy wall. Phys. Fluids. 9, 2429.

Barros, J. M. \& Christensen, K. T. 2014 Observations of turbulent secondary flows in a rough-wall boundary layer. J. Fluid Mech. 748, R1.

Bhaganagar, K, Kim, J \& Coleman, G 2004 Effect of roughness on wall-bounded turbulence. Flow Turbul. Combust. 72, 463-492.

Bradshaw, P. 1987 Turbulent secondary flows. Annu. Rev. Fluid Mech. 19, 53-74.

Chan, L., MacDonald, M., Chung, D., Hutchins, N. \& Ooi, A. 2015 A systematic investigation of roughness height and wavelength in turbulent pipe flow in the transitionally rough regime. J. Fluid Mech. 771, 743-777.

Chan, L., MacDonald, M., Chung, D., Hutchins, N. \& Ooi, A. 2018 Secondary motion in turbulent pipe flow with three-dimensional roughness. J. Fluid Mech. 854, 5-53.

Chung, D., Monty, J. \& Hutchins, N. 2018 Similarity and structure of wall turbulence with lateral wall shear stress variations. J. Fluid Mech. 847, 591-613.

Clauser, F. H. 1954 Turbulent boundary layers in adverse pressure gradients. J. Aeronaut Sci. 21, 91-108.

Flack, K. A., Schultz, M. P. \& Barros, J. M. 2020 Skin friction measurements of a systematically-varied roughness: probing the role of roughness amplitude and skewness. Flow Turbul. Combust. 104, 317 - 329.

Flack, K A, Schultz, M P \& Connelly, J S 2007 Examination of a critical roughness height for outer layer similarity. Phys. Fluids. 19, 095104.

Flack, K. A., Schultz, M. P. \& Shapiro, T. A. 2005 Experimental support for townsend's reynolds number similarity hypothesis on rough wall. Phys. Fluids. 17, 035102.

Forooghi, P., Stroh, A., Magagnato, F., Jakirlić, S. \& Frohnapfel, B. 2017 Toward a universal roughness correlation. J. Fluids Eng. 139, 121201.

Forooghi, P., Weidenlener, A., Magagnato, F., Böhm, B., Kubach, H., Koch, T. \& FroHNAPFEL, B. 2018 DNS of momentum and heat transfer over rough surfaces based on realistic combustion chamber deposit geometries. Int. J. Heat Fluid Flow. 69, 83-94.

Hama, F. R. 1954 Boundary-layer characteristics for smooth and rough surfaces. Trans. Soc. Nav. Archit. Mar. Engrs 62, 333-358.

Hamed, A M, Kamdar, A, Castillo, L \& Chamorro, L P 2015 Turbulent boundary layer over 2d and 3d large-scale wavy walls. Phys. Fluids. 27, 106601.

Hinze, J. O. 1967 Secondary currents in wall turbulence. Phys. Fluids. 10, S122S125.

Hudson, J D, Dykhno, L \& Hanratty, T J 1996 Turbulence production in flow over a wavy wall. Exp. Fluids. 20, 257-265.

Hutchins, N., Nickels, T. B., Marusic, I. \& Chong, M. S. 2009 Hot-wire spatial resolution issues in wall-bounded turbulence. J. Fluid Mech. 635, 103-136.

Hutchins, N., P., J., Nugroho, B., Ganapathisubramani, B. \& Utama, I. K. A. P 2016 
Turbulent boundary layers developing over rough surfaces: from the laboratory to fullscale systems. In Proceedings of 20th Australasian Fluid Mech. Conf. Perth, Australia.

Jelly, T. O. \& Busse, A. 2019 Reynolds number dependence of Reynolds and dispersive stresses in turbulent channel flow past irregular near-gaussian roughness. Int. J. Heat Fluid Flow. 80, 108485.

Jiménez, J. 2004 Turbulent flows over rough walls. Annu. Rev. Fluid Mech. 36, 173-196.

Kline, S. J., Reynolds, W. C., Schraub, F. A. \& Rundstadler, P. W. 1967 The structure of turbulent boundary layers. J. Fluid Mech. 30, 741-773.

Kruse, N, , Gunther, A \& Rudolf von Rohr, P 2003 Dynamics of large-scale structures in turbulent flow over a wavy wall. J. Fluid Mech. 485, 87-96.

Kruse, N, Kuhn, S \& Rohr, P R V 2006 Wavy wall effects on turbulence production and large-scale modes. J. Turbul. $\mathbf{7 ( 3 1 )}$.

Ligrani, P. M. \& BradshaW, P. 1987 Spatial resolution and measurement of turbulence in the viscous sublayer using subminiature hot-wires probes. Exp. Fluids. 5, 407-417.

Marusic, I., Monty, J. P., Hultmark, M. \& Smits, A. J. 2013 On the logarithmic region in wall turbulence. J. Fluid Mech. 716, R3, 1-11.

Medjnoun, T., Vanderwel, C. \& Ganapathisubramani, B. 2018 Characteristics of turbulent boundary layers over smooth surfaces with spanwise heterogeneities. J. Fluid Mech. 838, $516-543$.

Monty, J. P., Allen, J. J., Lien, K. \& Chong, M. S. 2011 Modification of the largescale features of high reynolds number wall turbulence by passive surface obtrusions. Exp. Fluids. 51, 1755-1763.

Monty, J. P., Dogan, E., Hanson, R., Scardino, A. J., Ganapathisubramani, G. \& Hutchins, N. 2016 An assessment of the ship drag penalty arising from light calcareous tubeworm fouling. Biofouling. 32(4), 451-464.

Morgan, J. \& MCKeOn, B.J. 2018 Relation between a singly-periodic roughness geometry and spatio-temporal turbulence characteristics. Int. J. Heat Fluid Flow. 71, $322-333$

Nakagawa, S., Na, Y \& Hanratty, T. J. 2003 Influence of a wavy boundary on turbulence. i. highly rough surface. Exp. Fluids. 35, 422-436.

Nakato, M, Onogi, H, Himeno, Y, Tanaka, I \& Suzuki, T 1985 Resistance due to surface roughness. Proceedings of the 15th Symposium on Naval Hydrodynamics pp. 553-568.

Napoli, E., Armenio, V. \& De Marchis, M. 2008 The effect of the slope of irregularly distributed roughness elements on turbulkent wall-bounded flows. J. Fluid Mech.613, 385-394.

NikURADSE, J. 1933 Strömungsgesetze in rauhen rohren. VDI-Forsch $\mathbf{3 6 1}$.

Nugroho, B., Hutchins, N. \& Monty, J. P. 2013 Large-scale spanwise periodicity in a turbulent boundary layer induced by highly ordered and directional surface roughness. Int. J. Heat Fluid Flow. 41, 90- 102.

Perry, A. E. 1982 Hot-wire anemometry. Oxford UK,: Oxford University Press.

Perry, A E \& Li, J D 1990 Experimental support for the attached-eddy hypothesis in zeropressure-gradient turbulent boundary layers. J. Fluid Mech. 218, 405-438.

Perry, A. E., Schofield, W. H. \& Joubert, P. N. 1969 Rough wall turbulent boundary layers. J. Fluid Mech. 37, 383-413.

Reda, C J, Ketter, J \& C, FAn 1974 Compressible turbulent skin friction on rough and rough/wavy walls in adiabatic flow. 7th Fluid and Plasma Dynamics Conference.

Reynolds, W. C. \& Hussain, A. K. M. F. 1972 The mechanics of an organized wave in turbulent shear flow. part 3. theoretical models and comparisons with experiments. $J$. Fluid Mech. 54, 263-288.

Schultz, M. P. \& FlaCK, K. A. 2005 Outer layer similarity in fully rough turbulent boundary layers. Exp. Fluids. 38, 328-340.

Schultz, M. P. \& Flack, K. A. 2007 The rough-wall turbulent boundary layer from the hydraulically smooth to the fully rough regime. J. Fluid Mech. 580, 381-405.

Schultz, M. P. \& Flack, K. A. 2009 Turbulent boundary layers on a systematically varied rough wall. Phys. Fluids. 21, 015104.

Sun, Z, Zhu, Y, Hu, Y \& Zhang, S 2018 Direct numerical simulation of a fully developed compressible wall turbulence over a wavy wall. J. Turbul. 19(1), 72-105.

Talluru, K. M., Kulandaivelu, V., Hutchins, N. \& Marusic, I. 2014 A calibration 
technique to correct sensor drift issues in hot-wire anemometry. Meas Sci Technol. 25, 105304.

Townsend, A. A. 1976 The structure of turbulent shear flow, 2nd ed. Cambridge University Press.

Wangsawijaya, D. D., Baidya, R., Chung, D., Marusic, I. \& Hutchins, N. 2020 The effect of spanwise wavelength of surface heterogeneity on turbulent secondary flows. $J$. Fluid Mech. 894, A7.

Willingham, D., Anderson, W., Christensen, K. T. \& Barros, J. M. 2014 Turbulent boundary layer flow over transverse aerodynamic roughness transitions: induced mixing and flow characterization. Phys. Fluids. 26, 025111.

YANG, J. \& ANDERSON, W. 2018 Numerical study of turbulent channel flow over surfaces with variable spanwise heterogeneities: Topographically-driven secondary flows affect outerlayer similarity of turbulent length scales. Flow Turbul. Combust. 100:1, 1-17.

Zenklusen, A, Kuhn, S \& Rudolf von Rohr, P 2012 Structural dissimilarity of large-scale structures in turbulent flows over wavy walls. Exp. Fluids. 24, 055112.

Zilker, D P, Cook, G W \& Hanratty, T J 1977 Influence of the amplitude of a solid wavy wall on a turbulent flow. part 1. non-separated flows. J. Fluid Mech. 82(1), 29-51. 